\title{
High birefringence photonic crystal fiber with a complex unit cell of asymmetric elliptical air hole cladding
}

\author{
Yuh-Sien Sun, ${ }^{1}$ Yuan-Fong Chau, ${ }^{1, *}$ Han-Hsuan Yeh, ${ }^{1}$ Lin-Fang Shen, ${ }^{2}$ \\ Tzong-Jer Yang, ${ }^{3}$ and Din Ping Tsai ${ }^{4}$ \\ ${ }^{1}$ Department of Electronic Engineering, Ching Yun University, Jung-Li, Taiwan, China \\ 2Department of Information and Electronic Engineering, Zhejiang University, Hangzhou 310027, China \\ ${ }^{3}$ Department of Electrophysics, National Chiao-Tung University, HsinChu 300, Taiwan, China \\ ${ }^{4}$ Department of Physics and Center for Nanostorage Research, National Taiwan University, Taipei, Taiwan, China \\ *Corresponding author: yfc01@cyu.edu.tw
}

Received 24 January 2007; revised 11 March 2007; accepted 12 March 2007;

posted 13 March 2007 (Doc. ID 79303); published 9 July 2007

\begin{abstract}
High birefringence induced by elliptical air hole photonic crystal fibers (EHPCFs) is analyzed numerically using the finite-element method. Statistical correlations between the birefringence and the various parameters are obtained. We found that the complex elliptical air hole is better than that of a circular one to obtain high birefringence in photonic crystal fibers. Our suggested structures can considerably enhance the birefringence in EHPCFs and show that the birefringence can be as high as $1.1294 \times 10^{-2}$, which is higher than the birefringence obtained from conventional step-index fiber $\left(5 \times 10^{-4}\right)$, circular air holes PCF $\left(3.7 \times 10^{-3}\right)$, and elliptical hollow PCF $\left(2.35 \times 10^{-3}\right)$. C 2007 Optical Society of America

OCIS codes: $060.2400,060.2280$.
\end{abstract}

\section{Introduction}

Photonic crystal fibers (PCFs) guide the electromagnetic field by an arrangement of air holes that run down the entire fiber length. In the holey fibers, the air holes reduce the average index around the solid core, and the guidance can be ascribed to the total internal reflection $[1,2]$. Recently, the most common cladding of PCFs has been investigated extensively [3-6], which consists of circular air holes, arranged in a triangular lattice with symmetric structure. For any fibers with rotational symmetry of an order higher than 2, a mode that has a preferred direction must be one of a pair of degenerate modes. Symmetry in PCFs implies the existence of doubly-degenerate pairs of modes, that share the same propagation constant $(\beta)$ and free-space wavelength $(\lambda)$, similar to the uniformly polarized $\mathrm{HE}_{11}$ modes in a conventional step-index fiber, so they must be degenerate. When this symmetry in PCFs is affected by some factors, such as bends and twists, stresses, or by manufacturing imperfections, the degeneracy is lifted and the

0003-6935/07/225276-06\$15.00/0

(C) 2007 Optical Society of America real parts of the effective indices $\left(n_{\text {eff }} \equiv \lambda \beta / 2 \pi\right)$ of the degenerate modes separate by an amount termed modal birefringence. As a result, the observation of birefringence must be a result of asymmetry in the structure. These perturbations couple the modes that propagate at slightly different phase velocities, with the consequence that the polarization of light becomes unpredictable after a short propagation.

When control of the polarized light is important, high birefringence as high as $5 \times 10^{-4}$ can be induced in conventional fibers [4,7], and this reduces the coupling between the once degenerate modes. Recently, several studies show that PCFs could exhibit birefringence of $\sim 1$ order of magnitude higher than that obtained from conventional techniques [8-10]. High birefringence fibers have been widely used for polarization control in fiber-optic sensors, precision optical instruments, and optical communication systems $[11,12]$. High birefringence in optical fibers has been usually achieved either by asymmetric shape in waveguide, such as an elliptical core [13], or by asymmetric stress distribution around the core incorporating the stress applying arts which replace circular air holes in the cladding with elliptical ones $[8,14]$, or 
squeeze the lattice of air holes [15,16]. In recent high data rate optical communications, first-order polarization mode dispersion (PMD) compensation techniques requires polarization maintaining fibers to utilize its differential group delay between the two eigen polarization states $[17,18]$. To enhance the merit in PMD compensation, high birefringence, i.e., large differential group delay, is required along with a low propagation loss.

Motivated by these previous works and addressing the challenges, we propose a novel design, to the best of our knowledge, of highly birefrigent index guiding photonic crystal fiber and it is composed of a solid silica core and a cladding with different sizes of complex (or binary) squeezed-triangular-lattice elliptical air holes. There are many methods to induce birefringence in PCFs. The key point is to destroy the symmetry of the structure and make the effective index difference between the two orthogonal polarization states. Due to the large contrast in the refractive index between the two orthogonal polarization states, high birefringence can be achieved with a relatively small index difference between the core and the cladding. The proposed structure that is used is a complex unit cell (two different sizes of elliptical air holes) to replace the single unit cell (one size of elliptical air hole) in the cladding region of the PCF. Owing to the gaps between two different air hole sizes of complex unit cell larger than that of single unit cell [17], the corresponding fiber structure designed by complex unit cell cladding is stronger in fiber strength than that designed by a single unit cell under external forces. The birefringence of our proposed structure contributes to the whole cladding asymmetry, unlike a similar structure with holes of different sizes used near the central region of PCF [19] and a structure with equal elliptical holes [16]. The fiber core is a point defect, which is formed by the omission of one smaller elliptical air hole in the center of this structure, in which the mode field is well confined in the core region; thus, it is possible to create a high birefringence PCF. The merit of a complex unit cell of different size elliptical air holes and its asymmetry could endow a new degree of freedom to design the birefringence in optical waveguides. In this paper, the origin of the birefringence is discussed, and its dependence on the structural parameters is analyzed numerically in the proposed elliptical air hole photonic crystal fibers (EHPCFs).

\section{Simulation Method}

The numerical method used in this paper is the finiteelement method (FEM), which is adequate for the analysis of general dielectric waveguide geometries. It has already already been successfully applied to investigate dispersion properties of triangular and cobweb PCFs [20,21]. The fiber cross-section representation is very accurate as the domain is divided into subdomains with triangular or quadrilateral shapes where any refractive index profiles can be properly represented. The PCFs we analyzed have elliptical holes, which have been successfully manu- factured recently [22]. Calculations have been done using the FEM [20,21,23, and 24], in which this method is described in detail and its convergence and suitability for computing the birefringence in arbitrarily shaped scatterers are also assessed. To modelize infinite PCF with a two-dimensional-finite-geometry model (i.e., to enclose the computational domain without affecting the numerical solution), it is necessary to use an anisotropic perfectly matched layers (PMLs), which are placed before the outer boundary.

\section{Simulation Models, Results, and Discussions}

Together with the technological advancement in the fabrication of PCFs $[14,16,22-24]$, it is possible to fabricate our suggested EHPCF. The structure of our proposed EHPCF used in this study is shown in Fig. 1(b). This design is different from the previous circular air holes PCF designed in [25] (we name it previous structure throughout this paper), as shown in Fig. 1(a), whereas, the radius of small and large circular air holes are $a$ and $b$, respectively. In our proposed structure, each lattice point forms the cladding which consists of complex (or binary) unit cell (two different sizes of elliptical air holes) with a pitch (center-to-center distance between the holes), $\Lambda=$ $1.96 \mu \mathrm{m}$. As shown in Fig. 2(b), $r_{1}$ and $r_{2}$ denote the half-length of the large elliptical holes along $x$ (short axis) and $y$ (major axis) directions, respectively. In the same manner, $r_{3}$ and $r_{4}$ represent the smaller elliptical ones. The core is formed by the omission of one small air hole in the centers of such structures. A point defect is created in this complex triangle lattice to form the PCF. We also define a coefficient $\eta=$ $a / b=r_{1} / r_{3}$ to determine the relationship between the large and small air hole sizes. In addition, a solid silica jacket was added to increase the diameter and strength of this fiber [26]. The refractive index of the background silica is set as $n=1.45$.

In Fig. 1(b), birefringence is introduced by the difference between two orthogonal directions. In the $x$ and $y$ directions of two different linear, homogeneous, and isotropic dielectrics, Maxwell's equations impose the boundary conditions for the normal and the transverse components of magnetic fields. One important fact is that the mode fields in EHPCFs looks

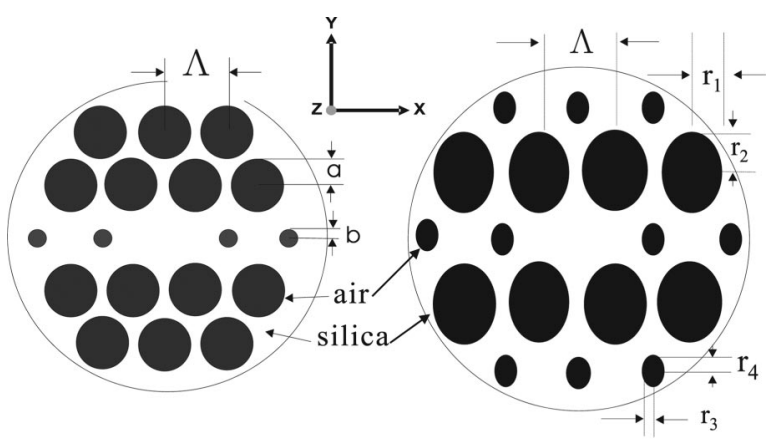

(a)

(b)

Fig. 1. PCF structure. (a) Previous structure (PCF structure designed in Ref. 2) and (b) our proposed EHPCF structure. 


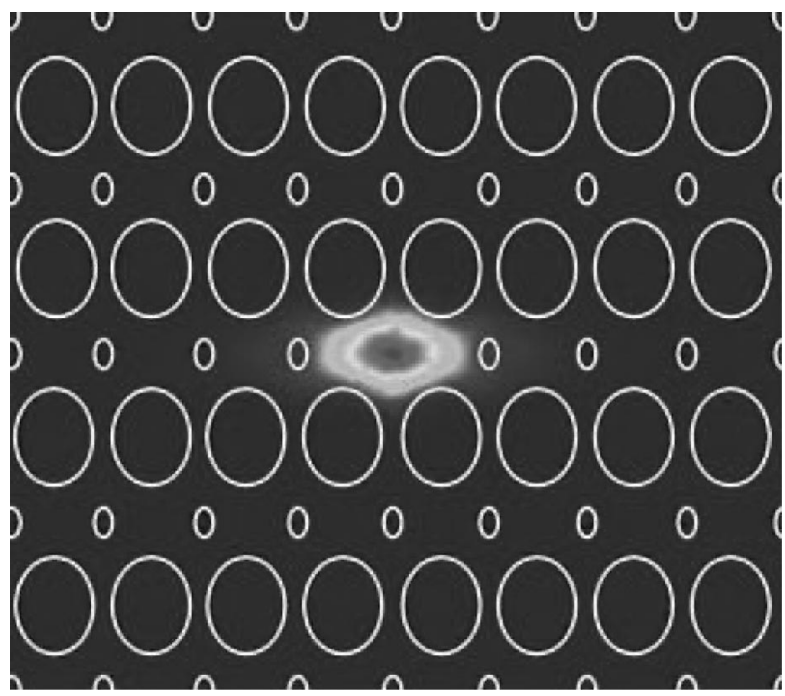

Fig. 2. (Color online) Mode field pattern with parameters, $\Lambda=$ $1.96 \mu \mathrm{m}, \quad r_{1}=0.9 \mu \mathrm{m}, r_{2}=1.084 \mu \mathrm{m}, r_{3}=0.18 \mu \mathrm{m}, r_{4}$ $=0.22 \mu \mathrm{m}, \eta\left(r_{1} / r_{3}\right)=5$ and elliptical ratio $r_{1} / r_{2}=r_{3} / r_{4}=0.83$ at excitation wavelength $\lambda=1.55 \mu \mathrm{m}$.

like the $\mathrm{HE}_{11}$ mode in step-index fibers, which we refer to as $\mathrm{HE}_{11 x}$ and $\mathrm{HE}_{11 y}$. The doublet components of the fundamental mode, as we know, are degenerate in conventional standard fibers and in circular holes triangular lattice PCFs. However, when the air holes are elliptical, the degeneracy splits significantly. Regardless of whether both modes are confined or leaky, the birefringence will be defined as $\Delta n=\mid n_{\text {eff }}^{x}-$ $n_{\text {eff }}^{y} \mid$, where $n_{\text {eff }}^{x}$ and $n_{\text {eff }}^{y}$ are the refractive indices of the $x$ - and $y$-polarization modes, respectively. To illustrate the field profile of our proposed EHPCF, the fundamental mode of our designed fiber with the parameters, $\Lambda=1.96 \mu \mathrm{m}, r_{1}=0.9 \mu \mathrm{m}, r_{2}=$ $1.084 \mu \mathrm{m}, r_{3}=0.18 \mu \mathrm{m}, r_{4}=0.22 \mu \mathrm{m}, \eta\left(r_{1} / r_{3}\right)=5$, and elliptical ratio $r_{1} / r_{2}=r_{3} / r_{4}=0.83$ at excitation wavelength $\lambda=1.55 \mu \mathrm{m}$, is shown in Fig. 2. The simulated results of the $y$ - and $x$-polarized mode are strongly bounded in the high index core region with the effective index $n_{\text {eff }}^{x}=1.388207$ and $n_{\text {eff }}^{y}=$ 1.380276, respectively, giving a birefringence $\Delta n=$ $\left|n_{\text {eff }}^{x}-n_{\text {eff }}^{y}\right|=7.931 \times 10^{-3}$, which is much higher than the structure as shown in [25]. It is evident in Fig. 2 that the intensity of the $x$-polarized mode is stronger than that of the $y$ polarized due to the $x$-polarized states have the lower air filling fraction than the $y$ polarized states. It implies that the asymmetry in EHPCFs is one of the key factors in determining the localization extent of the transverse mode. Another significant result from Fig. 2 is that the asymmetric core shape can influence the polarization mode in EHPCFs and can split the fields extended far beyond the core-cladding interface. It is noteworthy to confirm that if the large birefringence is desired, the parameters in EHPCFs are limited by the requirement for mode profiles, which contain higher fields intensity in the core region.

The effective index and birefringence of PCFs are relative to the air hole sizes and varying wavelength

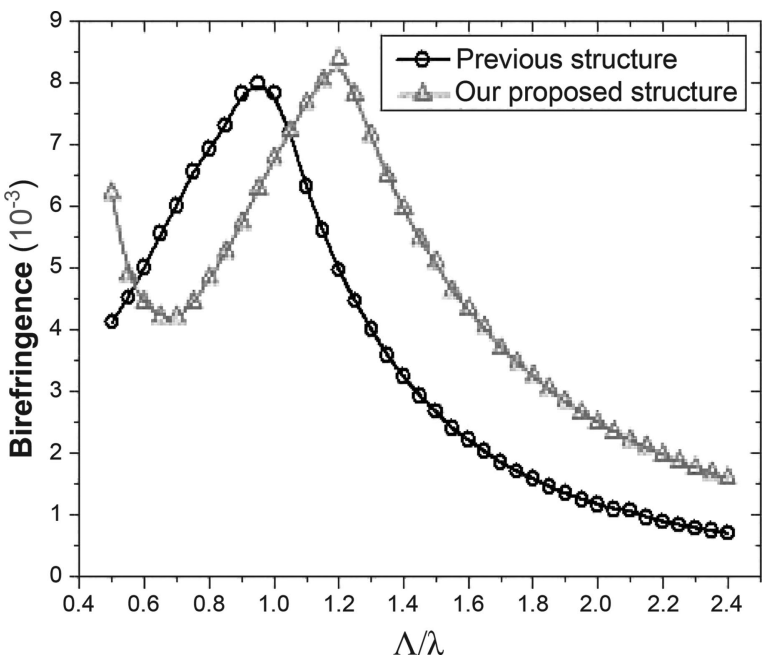

Fig. 3. (Color online) Birefringence as a function of normalized frequency $\Lambda / \lambda$, whereas the hole spacing $\Lambda=1.96 \mu \mathrm{m}$, the ratio of large and small air hole $\eta=a / b=r_{1} / r_{3}=5.5$.

(or normalized frequency $\Lambda / \lambda$ ). As expected, the difference between two polarized direction modes in cladding asymmetry can cause high birefringence in EHPCFs. Figure 3 shows the birefringence as a function of normalized frequency $\Lambda / \lambda$, whereas the hole spacing remains constant at $\Lambda=1.96 \mu \mathrm{m}$, the ratio of large and small air hole fixed $\eta=a / b=r_{1} / r_{3}$ $=5.5$. It can be clearly seen that the birefringence is sensitive to the varying wavelength $\lambda$, and the birefringence in our proposed structure is higher than that of previous structures when the value of $\Lambda / \lambda$ is larger than 1.05. Namely, the wavelength is also one of the key factors in determining the localization extent of the transverse mode. In Fig. 3, the birefringence reaches its maximum value $\Delta n=8.732 \times$ $10^{-3}$ at $\Lambda / \lambda=1.2$, and the birefringence decreases as the value of $\Lambda / \lambda$ is higher than 1.2. The corresponding wavelength $\lambda=1.55 \mu \mathrm{m}$ occurs at the value of $\Lambda / \lambda=1.25$ with the birefringence $\Delta n=7.792$ $\times 10^{-3}$ for our proposed structure, and $\Delta n=4.461$ $\times 10^{-3}$ for previous structures. It is evident in Fig. 3 that the field is confined in the high-index core at smaller $\Lambda / \lambda$ (longer wavelengths) and the field penetrates further into the asymmetry cladding region at larger $\Lambda / \lambda$ (shorter wavelengths).

The asymmetry as well as the leakage (or loss) in EHPCFs due to the interruption of the lattice are evident. The field confinement and its decay rate play a fundamental role in the leakage properties. They depend on the air hole diameter, their pitch and the number of rings. In practice, six to ten rings of air holes are often needed to reduce the confinement loss to an acceptable level. For the sake of accuracy, we use 13 rings to demonstrate the performance of birefringence. In the following analysis, we maintain the wavelength $\lambda=1.55 \mu \mathrm{m}, \Lambda=1.96 \mu \mathrm{m}, r_{1} / r_{2}=$ $r_{3} / r_{4}=0.83, \eta=a / b=r_{1} / r_{3}=3$ and the number of rings $N=13$. The influence of proposed complex asymmetry cladding on the birefringence stability is also significantly, which is illustrated in Fig. 4. It can 


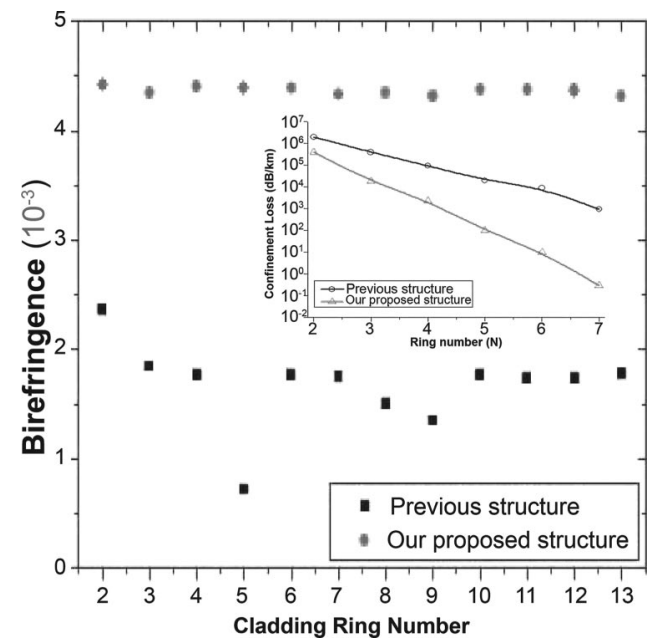

Fig. 4. (Color online) Birefringence as a function of the number of rings for $\lambda=1.55 \mu \mathrm{m}, \Lambda=1.96 \mu \mathrm{m}, r_{1} / r_{2}=r_{3} / r_{4}=0.83, \eta$ $=a / b=r_{1} / r_{3}=3$ and the number of rings $N=13$. Inset: the confinement loss as a function of the number of rings for $N=1-7$.

be clearly seen from Fig. 4 that the performance of birefringence of our proposed structure shown in Fig. $1(b)$ is more stable and higher than that of previous structures shown in Fig. 1(a), even in the case of the numbers of rings $N=13$. The birefringence of our designed structure tends to be stable, in the same manner, the birefringence in the previous structure shown in Fig. 1(b) quickly decreases when the numbers of rings larger is than $N=4$.

The FEM with PMLs which are placed before the outer boundary can be used to calculate the confinement loss of PCFs. The imaginary part of the complex effective index represents the loss. As described in Refs. 27 and 28, for small holes and few rings of holes, the confinement loss is huge, but decreases rapidly as the air hole diameter increases or more rings of holes are employed. The inset of Fig. 4 shows the confinement loss with fixed parameters, $\lambda=$ $1.55 \mu \mathrm{m}, \Lambda=1.96 \mu \mathrm{m}, r_{1} / r_{2}=r_{3} / r_{4}=0.83, \eta=$ $a / b=r_{1} / r_{3}=3$ and the number of rings is $N=7$ (because $N>7$ the confinement loss used FEM with PMLs in calculation is out of our computer memory). It can be seen in the inset of Fig. 4, the previous structure displays more confinement loss, whereas our proposed structure can reduce at least a magnitude of 1 order of confinement loss than that of the previous one. The confinement loss of our proposed structure is $\sim 0.39 \mathrm{~dB} / \mathrm{km}$, which is much lower than the value of about $\sim 490 \mathrm{~dB} / \mathrm{km}$ obtained from the previous structure when the air hole rings are $N=7$. To explain this phenomenon, the confinement field of our proposed structure is assigned to the PCF core, which is formed by the point defect and gives rise to more fields confined in the core region, thus the confinement loss decreases as more rings of holes are employed. In contrast, the small air holes array in the central part of the previous structure is a line defect, which demonstrates that most of the field leakage

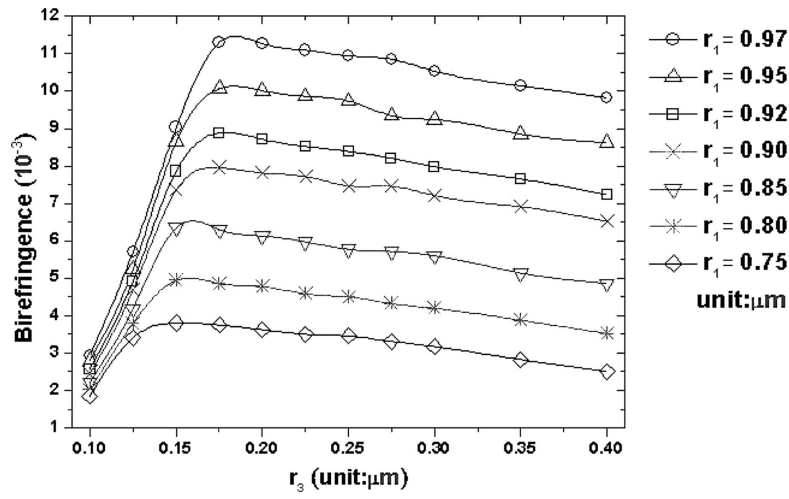

Fig. 5. Relationship between birefringence and the short axis of small elliptical holes $r_{3}$ (varying in the range of [0.1, 0.4] $\mu \mathrm{m}$ with a different short axis of large elliptical holes $r_{1}$. The parameters are maintained at $\Lambda=1.96 \mu \mathrm{m}$, elliptical ratio $r_{1} / r_{2}=r_{3} / r_{4}=0.83$ and the excitation wavelength $\lambda=1.55 \mu \mathrm{m}$.

comes from the $x$ direction, and higher confinement loss is presented.

Figure 5 shows the relationship between birefringence and the short axis of small elliptical holes $r_{3}$ (varying in the range of $[0.1,0.4] \mu \mathrm{m}$ with different short axis of large elliptical holes $r_{1}$ ). The parameters are maintained at $\Lambda=1.96 \mu \mathrm{m}$, elliptical ratio $r_{1} / r_{2}=r_{3} / r_{4}=0.83$ and the excitation wavelength $\lambda=1.55 \mu \mathrm{m}$. The results show that the birefringence increases rapidly as $r_{3}$ increases to a value of $0.175 \mu \mathrm{m}$. When $r_{3}$ is beyond $0.175 \mu \mathrm{m}$, the birefringence decays slowly as $r_{3}$ increases. If $r_{1}$ decreases when keeping $\Lambda$ constant, the values of birefringence decrease as the value of $r_{1}$ decreases. For instance, a value of birefringence $\Delta n=3.5 \times 10^{-3}$ is obtained for $r_{1}=0.75 \mu \mathrm{m}\left(r_{3}=0.175 \mu \mathrm{m}\right)$, which is higher than that of the elliptical hollow PCF obtained from Ref. 29 . In the results of our simulation, the high birefringence is mainly affected depending on the variation of large elliptical air holes. What is the physical reason for inducing high birefringence in EHPCFs? It can be expected that the average effective index $n_{\text {eff }}^{y}$ is reduced and the mode field is extended more to the cladding region as the area of large elliptical air holes along the $y$ axis increased. In contrast to the average effective index $n_{\text {eff }}^{x}$ in the core region, which is reduced less due to the area of small elliptical air holes along the $x$ axis can confine more field and contribute to a higher $n_{\text {eff }}^{x}$ value. This is why the high birefringence $\Delta n$, the mode index difference between the $\mathrm{HE}_{11 x}$ and the $\mathrm{HE}_{11 y}$ modes, i.e., $\Delta n=\left|n_{\text {eff }}^{x}-n_{\text {eff }}^{y}\right|$, can be obtained by determining the optimal parameters in our suggested EHPCFs. A maximum value of birefringence $\Delta n=1.1294 \times 10^{-2}$ is achieved for $r_{3}=$ $0.175 \mu \mathrm{m}\left(r_{1}=0.97 \mu \mathrm{m}\right)$, indicating that the birefringence of about an order of magnitude higher than that of value $\Delta n=3.7 \times 10^{-3}$ reported by the previous structure in Ref. [25].

Figure 6 shows the birefringence as a function of the short axis ratio $\eta\left(r_{1} / r_{3}\right)$ of elliptical hole at different elliptical ratios $\left(r_{1} / r_{2}=r_{3} / r_{4}\right)$ with structure parameters $\Lambda=1.96 \mu \mathrm{m}$ and the excitation wavelength 


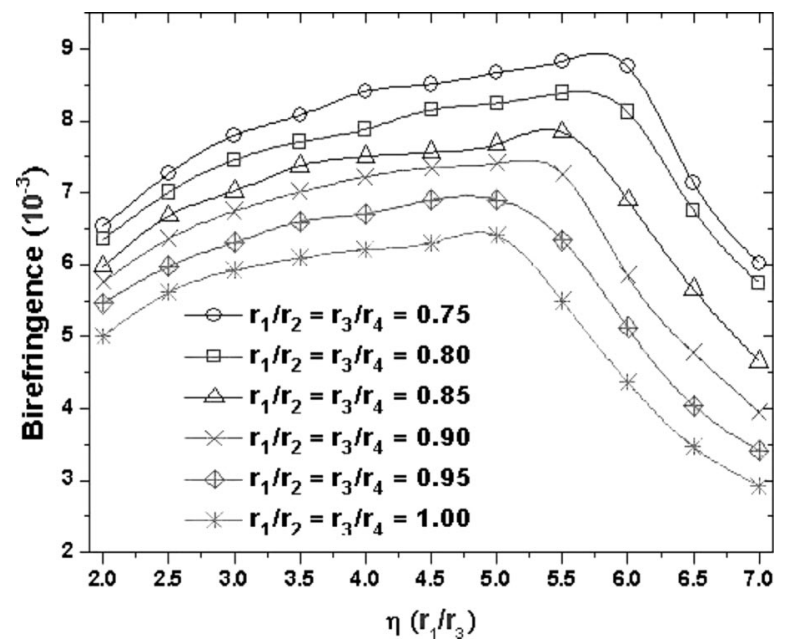

Fig. 6. Birefringence as a function of the short axis ratio $\eta\left(r_{1} / r_{3}\right)$ of elliptical hole at different elliptical ratios $\left(r_{1} / r_{2}=r_{3} / r_{4}\right)$ with structure parameters $\Lambda=1.96 \mu \mathrm{m}$ and the excitation wavelength $\lambda=1.55 \mu \mathrm{m}$.

$\lambda=1.55 \mu \mathrm{m}$. In Fig. 6, the elliptical ratio is varied from $r_{1} / r_{2}=r_{3} / r_{4}=0.75$ to 1 (the value 1 means the elliptical shape becomes circular) with the increment 0.05 . It can be clearly seen that the birefringence $\Delta n$ increases as the elliptical ratio decreases. Birefringence $\Delta n$ increases monotonically when the short axis ratio $\eta\left(r_{1} / r_{3}\right)$ of the elliptical hole increases. A maximum value $8.831 \times 10^{-3}$ is achieved at a fixed $\eta\left(r_{1} / r_{3}\right)=5.5$ when the elliptical ratio $r_{1} / r_{2}=r_{3} / r_{4}$ $=0.75,0.8$ and 0.85 . It can be concluded that the elliptical air hole is better than the circular air hole in obtaining the high birefringence in PCF. By comparing Figs. 5 and 6, one will find that the average effective index of the $x$ axis is higher than that of the $y$ axis.

Figure 7 shows the simulation results of comparing birefringence between previous structures and our proposed structure as a function of effective area of

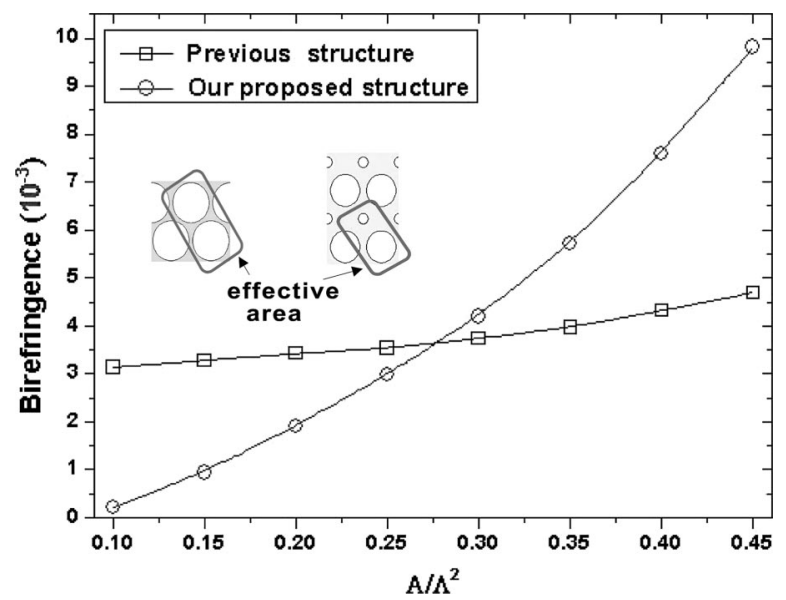

Fig. 7. Comparing the birefringence between the previous structure and our proposed structure as a function of the effective area of air holes $A / \Lambda^{2}$ in the cladding region with structure parameters $\Lambda=1.96 \mu \mathrm{m}, \eta\left(r_{1} / r_{3}\right)=5.5$, and the excitation wavelength $\lambda$ $=1.55 \mu \mathrm{m}$. air holes $A / \Lambda^{2}$ in the cladding region with structure parameters $\Lambda=1.96 \mu \mathrm{m}, \eta\left(r_{1} / r_{3}\right)=5.5$ and the excitation wavelength $\lambda=1.55 \mu \mathrm{m}$. The birefringence of our proposed structure increases quickly as the effective area $A / \Lambda^{2}$ increases. In contrast to the previous structure shown in Fig. 1(b), the birefringence is less sensitive to the variation of the effective area $A / \Lambda^{2}$. With the increasing effective area in our proposed EHPCFs cladding, the difference of the air filling fraction becomes large between the $x$ and $y$ directions; thus, high birefringence is obtained. As a consequence, the high birefringence increment corresponds to the increment of the effective area due to the increment of difference between $n_{\text {eff }}^{x}$ and $n_{\text {eff. In }}^{y}$ Fig. 7, the birefringence in the previous structure increases gradually and has a higher birefringence as the value of $A / \Lambda^{2}$ less than 0.276 , in which the birefringence of the previous structure and our proposed structure have the same birefringence of $3.68 \times$ $10^{-3}$. When $A / \Lambda^{2}$ is higher than 0.276 , the birefringence of our proposed structure is much higher than that of the previous structure. A maximum value of birefringence reaches $9.814 \times 10^{-3}$ corresponding to $A / \Lambda^{2}=0.45$, which is much higher than the value of $4.69 \times 10^{-3}$ obtained from the previous structure.

Similar to the conventional and elliptical hollow high birefringence fibers, the advantages of our proposed EHPCFs discussed above can be used for a number of applications [30-39] and allow for highly birefringent devices with much broader wavelength range to be created. In addition, the high birefringence EHPCFs, coupled with the use of endlessly single mode PCFs or endlessly single mode polarization maintaining PCFs as lead-in/lead-out fibers, would allow for extreme broadband devices that cannot be created with conventional technologies.

\section{Conclusion}

A highly birefringent index-guiding elliptical air holes PCFs with asymmetric cladding is successfully demonstrated. The birefringence is achieved by utilizing the intrinsically large index contrast in PCFs in combination with asymmetric cladding designs. The birefringence is found to be largely dependent on the variation of the normalized frequency, size ratio and effective area of the elliptical air holes, and it can be concluded that the complex elliptical air hole is better than that of circular one in obtaining the high birefringence in PCF. Our suggested structures can considerably enhance the birefringence in EHPCFs and show that the birefringence can be as high as $1.1364 \times 10^{-2}$, which is higher than those obtained from conventional step-index fiber $\left(5 \times 10^{-4}\right)$, ${ }^{1}$ circular air holes PCF $\left(3.7 \times 10^{-3}\right)$ [25] and elliptical hollow PCF $\left(2.35 \times 10^{-3}\right)$ [29]. There are many methods used to induce birefringence in PCFs. The key point is to destroy the symmetry of the structure, and make the effective index different between the two orthogonal polarization states. The suggested structure possesses a large birefringence to separate the two polarization modes. Our simulation results provide 
valuable insight into the realization of $\mathrm{PCF}$ with even higher birefringence than previously demonstrated in literature.

The authors acknowledge financial support from the National Science Council, Taiwan, Republic of China, under grants NSC 95-2112-M-231-001 and NSC-952119-M-009-029, and the Ministry of Economic Affairs, Taiwan, China, under grant 95-EC-17-A-08-S1-0006.

\section{References}

1. P. St. J. Russell, "Photonic crystal fibers," Science 299, 358 362 (2003).

2. A. M. Zheltikov, "Holey fibers," Phys. Usp. 170, 1203-1215 (2000).

3. P. R. McIsaac, "Symmetry-induced modal characteristics of uniform waveguides-I: summary of results," IEEE Trans. Microwave Theory Tech. 23, 421-429 (1975).

4. T. A. Birks, J. C. Knight, and P. St. J. Russell, "Endlessly single-mode photonic crystal fiber," Opt. Lett. 22, 961-963 (1997).

5. C. J. S. de Matos and J. R. Taylor, "Multi-kilowatt, all-fiber integrated chirped-pulse amplification system yielding 40 pulse compression using air-core fiber and conventional erbium-doped fiber amplifier," Opt. Express 12, 405-409 (2004).

6. F. Benabid, J. C. Knight, G. Antonopoulos, and P. St. J. Russell, "Stimulated Raman scattering in hydrogen-filled hollow-core photonic crystal fiber," Science 298, 399-402 (2002).

7. J. Noda, K. Okamoto, and Y. Sasaki, "Polarization-maintaining fibers and their applications," J. Lightwave Technol. 4, 10711089 (1986).

8. M. J. Steel and R. M. Osgood, "Polarization and dispersive properties of elliptical-hole photonic crystal fibers," J. Lightwave Technol. 19, 495-503 (2001).

9. K. Saitoh and M. Koshiba, "Photonic bandgap fibers with high birefringence," IEEE Photon. Technol. Lett. 14, 1291-1293 (2002).

10. A. Hochman and Y. Leviatan, "Modal dynamics in hollow-core photonic-crystal fibers with elliptical veins," Opt. Express 13, 6194-6201 (2005).

11. R. A. Bergh, H. C. Lefervre, and H. J. Shaw, "An overview of fiber-optic gyroscopes,” J. Lightwave Technol. 2, 91-107 (1984).

12. M. Nakazawa, "Highly efficient Raman amplification in a polarization-preserving optical fiber," Appl. Phys. Lett. 46, 628-630 (1985).

13. R. B. Dyott, J. R. Cozens, and D. G. Morris, "Preservation of polarization in optical-fiber waveguides with elliptical cores," Electron. Lett. 15, 380-382 (1979).

14. Z. Zhu and T. G. Brown, "Stress-induced birefringence in microstructured optical fibers," Opt. Lett. 28, 2306-2308 (2003).

15. T. Hosaka, K. Okamoto, T. Miya, Y. Sasaki, and T. Edahiro, "Low-loss single polarization fibers with asymmetrical strain birefringence," Electron. Lett. 17, 530-531 (1981).

16. K. Saitoh, "Single-polarization single-mode photonic crystal fibers," IEEE Photon. Technol. Lett. 18, 1243-1245 (2006).

17. L. Poti and A. Bogoni, "Experimental demonstration of a PMD compensator with a step control algorithm," IEEE Photon. Technol. Lett. 13, 1367-1369 (2001).

18. I. K. Hwang and Y. H. Lee, "High birefringence in elliptical hollow optical fiber," Opt. Express 12, 1916-1923 (2004).

19. X. Yu, M. Yan, L. W. Luo, and P. Shum, "Theoretical investigation of highly birefringent all-solid photonic bandgap fiber with elliptical claddrods," IEEE Photon. Technol. Lett. 15, 1384-1386 (2003).
20. Y. Yue, G. Kai, Z. Wang, T. Sun, L. Jin, Y. Lu, C. Zhang, J. Liu, Y. Li, S. Yuan, and X. Dong, "Highly birefringent elliptical-hole photonic crystal fiber with squeezed hexagonal lattice," Opt. Lett. 32, 469-471 (2007).

21. A. Cucinotta, S. Selleri, L. Vincetti, and M. Zoboli, "Holey fiber analysis through the finite element method," IEEE Photon. Technol. Lett. 14, 1530-1532 (2002).

22. N. A. Issa, M. A. Van-Eijkelenborg, M. Fellew, F. Cox, G. Henry, and M. C. J. Large, "Fabrication and study of microstructured optical fibers with elliptical holes," Opt. Lett. 29, 1336-1338 (2004).

23. S. Selleri, L. Vincetti, A. Cucinotta, and M. Zoboli, "Complex FEM modal solver of optical waveguides with PML boundary conditions," Opt. Quantum Electron. 33, 359-371 (2001).

24. D. Ferrarini, L. Vincetti, M. Zoboli, A. Cucinotta, and S. Selleri, "Leakage properties of photonic crystal fibers," Opt. Express 10, 1314-1319 (2002).

25. A. Ortigosa-Blanch, J. C. Knight, W. J. Wadsworth, J. Arriaga, B. J. Mangan, T. A. Birks, and P. St. J. Russell, "Highly birefringent photonic crystal fibers," Opt. Lett. 25, 1325-1327 (2000).

26. J. C. Knight, T. A. Birks, P. St. J. Ressel, and D. M. Atkin, "All-sillica single-mode optical fiber with photonic crystal cladding," Opt. Lett. 21, 1547-1549 (1996).

27. T. P. White, R. C. McPhedran, C. M. de Sterke, L. C. Botten, and M. J. Steel, "Confinement losses in microstructured optical fibers," Opt. Lett. 26, 1660-1662 (2001).

28. A. Betourne, V. Pureur, G. Bouwmans, Y. Quiquempois, L. Bigot, M. Perrin, and M. Douay, "Solid photonic bandgap fiber assisted by an extra air-clad structure for low-loss operation around $1.5 \mu \mathrm{m}$," Opt. Express 15, 316-324 (2007).

29. Y. Jung, S. R. Han, S. Kim, U. C. Paek, and K. Oh, "Versatile control of geometric birefringence in elliptical hollow optical fiber," Opt. Lett. 31, 2681-2683 (2006).

30. B. Y. Kim, J. N. Blake, S. Y. Huang, and H. J. Shaw, "Use of highly elliptical core fibers for two-mode fiber devices," Opt. Lett. 12, 729-731 (1987).

31. J. N. Blake, B. Y. Kim, and H. J. Shaw, "Fiber-optic modal coupler using periodic microbending," Opt. Lett. 11, 177-179 (1986).

32. W. V. Sorin, B. Y. Kim, and H. J. Shaw, "Highly selective evanescent modal filter for two-mode optical fibers," Opt. Lett. 11, 581-583 (1986).

33. B. Y. Kim, J. N. Blake, H. E. Engan, and H. J. Shaw, "All-fiber acousto-optic frequency shifter," Opt. Lett. 11, 389-391 (1986).

34. A. M. Vengsarkar, W. C. Michie, L. Jankovic, B. Culshaw, and R. O. Claus, "Fiber-optic dual-temperature sensor for simultaneous measurement of strain and temperature," J. Lightwave Technol. 12, 170-177 (1994).

35. S. H. Yun, I. K. Hwang, and B. Y. Kim, "All-fiber tunable and laser based on two-mode fiber," Opt. Lett. 21, 27-29 (1996).

36. H. S. Park, K. Y. Song, S. H. Yun, and B. Y. Kim, "All-fiber wavelength-tunable acoustooptic switches based on intermodal coupling in fibers," J. Lightwave Technol. 20, 18641868 (2002).

37. J. N. Blake, S. Y. Huang, B. Y. Kim, and H. J. Shaw, "Strain effects on highly elliptical core two-mode fibers," Opt. Lett. 12, 732-734 (1987).

38. S. Y. Huang, J. N. Blake, and B. Y. Kim, "Perturbation effects on mode propagation in highly elliptical core two-mode fibers," J. Lightwave Technol. 8, 23-33 (1990).

39. L. Provino, J. M. Dudley, H. Maillotte, N. Grossard, R. S. Windeler, and B. J. Eggleton, "Compact broadband continuum source based on microchip laser pumped microstructured fiber," Electron. Lett. 37, 558-560 (2001). 